\title{
Алгоритмическое и Программное Обеспечения для Выбора и Оценки Инновационного Проекта
}

\author{
Тарана Тагиева ${ }^{1}$, Шафагат Рагимов ${ }^{2}$, Джаваншир Мамедов ${ }^{3}$ \\ 1,2,3 Сумгаитский Государственный Университет, г. Сумгаит, Азербайджан \\ 1,2,3 cavan62@mail.ru
}

\begin{abstract}
Аннотация- На основе применения поэтапной схемы решения задачи предложен алгоритм управления инновационными проектами в научно-производственном центре технологического парка при высшем учебном заведении. Для экспертного анализа, поиска и выбора наилучшего инновационного проекта предложено математическое обеспечение. С помощью алгоритмической и математической модели разработана программа автоматизированного анализа, выбора и оценки инновационного проекта для дальнейшего его производственного выпуска в технологическом парке.
\end{abstract}

Ключевые слова- инновация, проект, управление, производственный центр, программное обеспечение

\section{I. АНАЛИЗ ВОПРОСА ВЫБОРА ИННОВАЦИОННЫХ ПРОЕКТОВ ДЛЯ ТЕХНОЛОГИЧЕСКОГО ПАРКА, ПОСТАНОВКА ЗАДАЧИ И ЦЕЛИ}

Разработка новых инновационных проектов в научноисследовательских отделах является сложным, многоэтапным научно-исследовательским процессом с применением теоретических методов и практического применения инженерных технологий, экспериментальных и производственных внедрений, а также их экономической оценки $[1,2]$. Традиционные научноисследовательские и производственные центры в европейских, азиатских и американских странах решают подобную проблему путем управления проектами с корпоративными связями между различными отделами исследовательского центра. Анализ существующих методов разработки новых инновационных проектов [3] показал, что в этих работах недостаточно решен вопрос их поиска и выбора на основе алгоритмического и программного обеспечения, которые обеспечили бы высокую экономическую эффективность и получение высоких финансовых результатов в бизнесе научноисследовательского и производственного центра. В связи с этим, были определены вопросы исследования по следующим направлениям:

1. Создание новой концепции управления инновационными проектами в научно-исследовательском и производственном центре технологического парка, который позволил бы выбрать и реализовать лучший инновационный проект в короткий промежуток времени;

2. Разработка алгоритмического и программного средства для обеспечения управления и оценки инновационными проектами в научно-исследовательском отделе технологического парка, которые могут быть предоставлены для выполнения программных процедур в корпоративной сети пользователей научноисследовательского производственного центра.

В зависимости от определенных научных проблем определяется цель данной работы, где предусматривается необходимость разработки универсального алгоритма и программы, которая обеспечивало бы эффективный выбор предлагаемых проектов с помощью экспертной оценки и разработка нового инновационного проекта, относящийся различным научным профилям.

\section{II. АЛГОРИТМИЧЕСКОЕ ОБЕСПЕЧЕНИЕ УПРАВЛЕНИЯ ИННОВАЦИОННЫМИ ПРОЕКТАМИ В НАУЧНО-ПРОИЗВОДСТВЕННОМ ЦЕНТРЕ}

Функции отделов управления научнопроизводственного центра на базе кафедры «Информационные технологии и программирование» с разработкой и коммерциализацией инновационного проекта обеспечиваются с помощью алгоритмических процедур. Процедуры управления инновационного проекта от начала регистрации до его внедрения в производстве реализуются на базе следующих этапов:

1. Профессорско-преподавательский состав и студенты ВУЗ-а представляют свои идеи на начальном уровне системы регистрации. Сначала вводятся информации в одном из двух панелей "Регистрачия учителя " или "Регистрация студента". Пользователь системы вводит свои данные и информацию о самом проекте (название, цель проекта, краткая идея проекта - аннотация), которая сохраняется в панели "Аннотация" для последующего ознакомления данного проекта экспертом. Кроме того, на первом этапе для тщательного анализа в этой части системы, используется панель "Прототипь проекта", где сохраняются данные подобные проекту (конструкционные, технологические и другие необходимые данные).

2. В течение короткого времени разные специалисты автономно проверяют регистрационные данные пользователя и его аннотацию. Выбор оптимального инновационного проекта (ИП) осуществляется в условиях неопределенности с помощью нижеследующей формулы [4]:

$$
\begin{gathered}
\text { ИП } \text { опт } в \text { выб }=\left\{\text { ИП } ^ { j } \operatorname { m a x } _ { j } \left[(1-\infty) * \min _{i} S_{i j}+\alpha *\right.\right. \\
\left.\left.\max _{i} S_{i j}\right]\right\}(1)
\end{gathered}
$$


где $\alpha$-коэфициент оптимального ИП, принимающий значение от 0 до $1 ; S_{i j}$ - известные характеристики ИП, приведенные в справочных данных .

Если $\alpha=1$, то выбор альтернативного проекта осуществляется по правилу максимакс, при $\alpha=0-$ по правилу максимин. Значение математического ожидания альтернативного проекта ј является критерием, на основе которого осуществляется выбор. Оптимальный вариант проекта определяется по формуле [4]:

$$
\text { ИП } \text { опт_выб }=\left\{\mathrm{H}^{j} \mid \max _{j} \sum_{i=1}^{n} S_{i j} * P_{i}\right\}
$$

где $P i$ - вероятность наступления состояния і-й внешней среды.

Для решения задачи выбора набора новых инновационных проектов можно использовать метод ранжирования альтернатив с учетом различных критериев отбора, где каждый проект оценивается независимо друг от друга. Определяются множество технических и экономических показателей инновационного проекта $\left(\Pi_{\text {ип_i }}\right)$ и требований к ним [3]:

$$
\begin{aligned}
& T \Pi_{u n}=\left\{\mathrm{T \Pi}_{\text {ип_1 }}, \text { тп ип_2 } 2, \ldots, \mathrm{T \Pi}_{\text {ип_ } n}\right\} .
\end{aligned}
$$

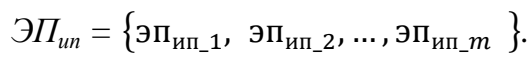

$$
\begin{aligned}
& T_{u n}=\left\{\mathbf{T}_{\text {ип_1 }}, \mathbf{T}_{\text {ип_2 }}, \ldots, \mathbf{T}_{\text {ип_n }}\right\} .
\end{aligned}
$$

Каждый технический и экономический показатель инновационного проекта $T \Pi_{u n j}$ и ЭП ипј ассоциирован с подмножеством требований к ним $\mathrm{T}_{\text {ип_ } i} \in \mathrm{T}_{\text {ип }}$, где і $a$ ? ? N $=\{1, \ldots, n\}$,

Задача выбора оптимального экономического показателя $\Pi_{\text {ип_i }}$ по стоимости совокупности проектов для реализации заданных требований целевая функция будет иметь следующий вид:

$$
F_{t r 1}(x)=\sum_{j=1}^{n} \mathrm{~T}_{\text {ипі }} x_{j} \rightarrow \min
$$

при условиях:

$$
\begin{gathered}
\sum_{j=1}^{n} a_{1 j} x_{j} \geq 1, \text { где } i=m \\
x \in 0,1, \text { где } j=n
\end{gathered}
$$

В этом случае задача структурного синтеза сводится определению экстремального значения целевой функции (3).

Если Э $\phi_{j}$ интерпретировать как эффективность проектов, то значение целевой функции определяет максимальную эффективность набора инновационных проектов, для построения системы, удовлетворяющей всем заданным требованиям при всех ограничениях (4) и (5):

$$
F_{t r 1}(x)=\sum_{j=1}^{n} \ni \phi_{j} x_{j} \rightarrow \max
$$

Введем в математическую модель данные о технических параметрах решаемых задач $\mathrm{R}$ путем добавления матрицы натуральных чисел В. Определим матрицу $B=\left(b_{i j}\right), \quad i \in m, j \in n$ :

$$
b_{i j}=\left\{\begin{array}{c}
b, \text { если } a_{i j}=1 \text { и } r_{i} \text { выполнено } \\
\text { объектом } j \text { с параметром } b=0 . .1 \\
0, \text { иначе }
\end{array}\right.
$$

Так как инновационные проекты имеют конкретные числовые характеристики, отражающие их количественные или качественные параметры по реализации функции (требования) $r_{j}$, то их можно взять в качестве величин $b_{i j}$, проведя стандартную процедуру согласования и нормализации (приведения к единому диапазону [0..1], (1 - лучший показатель, 0 - худший), чтобы их правомерно было сравнивать как показатели качества.

Процедуры эксперта выполняются и сохраняются в панели «Экспертиза».

3. Для получения тщательной презентации, проекты представляются экспертам в определенное предоставленное время. Новизна, современность, высокое инженерное решение и экономическая эффективность проектов являются основой выбора проекта для начального экспериментального исследования в лабораторных условиях в соответствии с научными профилями.

4. В экспериментальной лаборатории выбранный наилучший проект изготавливается в виде опытного образца, проверяются его технологические, конструкционные и функциональные характеристики. На этом этапе определяются основные технологические характеристики и уровень качества проекта, отличительные от данных прототипов.

5. Вся информация проектировщика сохраняется в базе данных («База данных Project»).

6. В коммерческом отделе определяются основные правила и требования на внешнем и внутреннем рынке, а также вычисляется экономическая эффективность проекта. Для представления проекта на местном и международном рынках в «Представление нового проекта» чертежи 2, 3-х - мерных изображений, анимации, видео и технические характеристики сохраняются в базе данных. Менеджеры по научным профилям, выбирая клиентов, сохраняют некоторые сведения о них в разделе «Клиент фирмы». С помощью этого радела между научнопроизводственным центром и фирмой осуществляется официальная встреча, где подготавливается пакет проектных документов.

7. На этом этапе все документы, проверенные экспертом направляются в гибкое производство, где осуществляется процесс изготовления проекта.

Представленный алгоритм с процедурами регистрации идеи и аннотации проекта; организации базы данных проекта; экспертной проверки и оценки проекта позволяет разработать системное программное обеспечение экспертного анализа и выбора инновационного проекта. 


\section{III. ПРОГРАММНОЕ ОБЕСПЕЧЕНИЕ АВТОМАТИЗАЦИИ ПРОЦЕССА АНАЛИЗА, ВЫБОРА И ОЦЕНКИ ИННОВАЦИОННОГО ПРОЕКТА}

Модуль формирования научной базы данных инновационных проектов обеспечивается путем сохранения всех данных об инновационном проекте в базе данных. В базе данных в виде таблицы структурируются данные в соответствии с областью применения. Для оценки каждого проекта в качестве инновационного, данные сохраняются в системе, а эксперт по соответствующей области проверяет инновационный проект и дает оценку по его разработке. Информация об оценке проекта направляется автору проекта. В этом сообщении ответ может обобщаться следующими версиями: принято; принято, но должно быть выполнены некоторые исправления; отвергнуто.

В соответствии функциям каждого модуля, для экспертного выбора и оценки инновационного проекта в корпоративной сети технологического парка разработана программа со следующими этапами (рисунок 1):

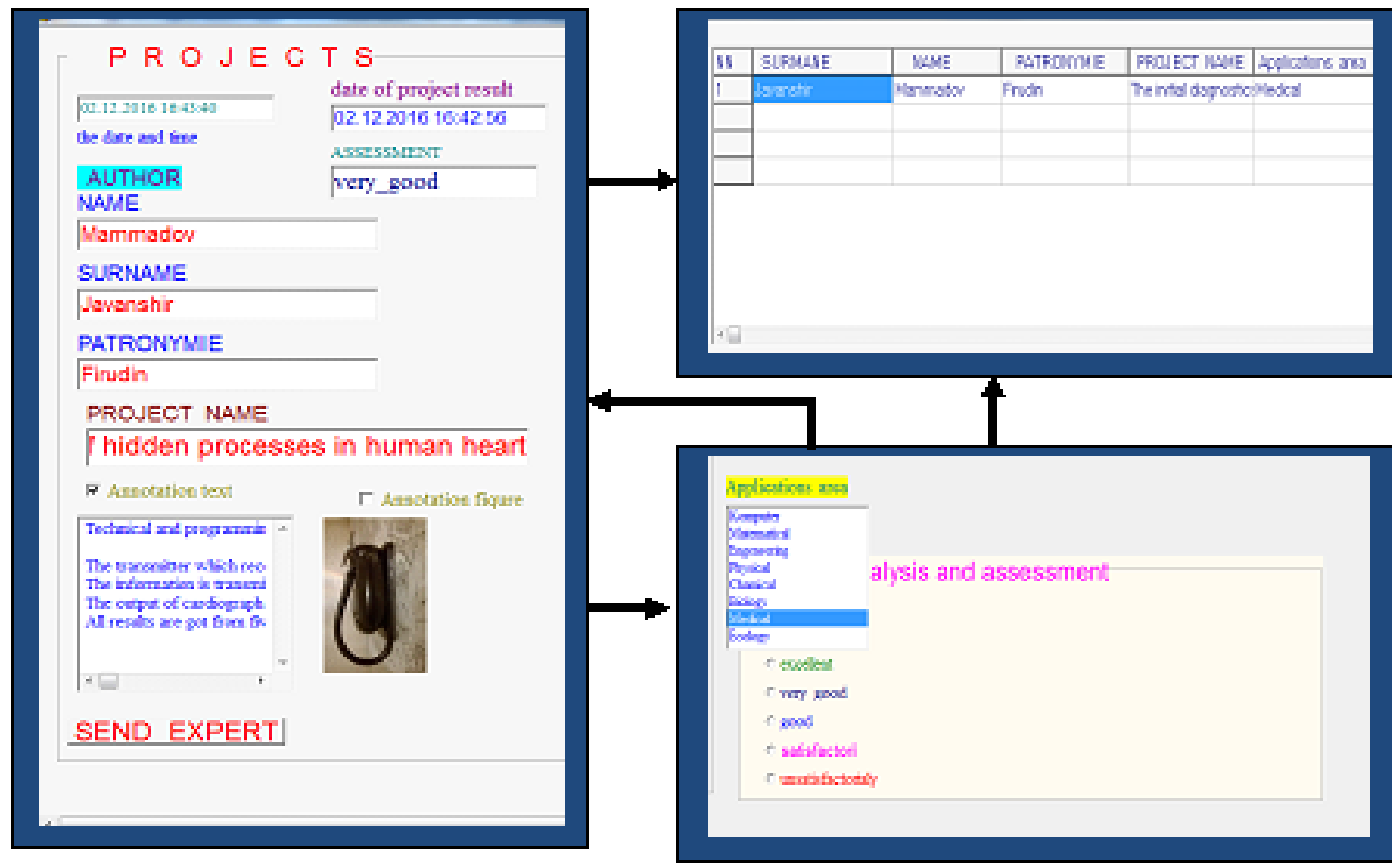

Рисунок. 1. Программное обеспечение выбора и оценки инновационного проекта в технологическом парке

1. На первом этапе проектировщик вводит персональные данные об авторах, название и цель проекта, аннотацию проекта, дата и время приема проекта в систему;

2. На втором этапе все данные по проекту сохраняются в системе управления базой данных в виде таблицы;

3. На третьем этапе эксперт проверяет информацию о конструкторе проекта и рассматривает аннотацию проекта. После проверки проекта дается экспертная оценка, итоги которых посылаются конструктору.

\section{ЗАКЛЮЧЕНИЕ}

На основании исследования управления инновационными проектами получены нижеследующие результаты:

1. В соответствии с целью и задачами исследования была предложена алгоритмическая процедура управления и оценки инновационного проекта в технопарке;

2. Разработано алгоритмическое обеспечение выбора альтернативного проекта, формирования базы данных проектов и оценка проекта, проведенного экспертом; 
3. На основе алгоритмических процедур управления была разработана программа для экспертной оценки инновационного проекта.

\section{ЛИТЕРАТУРА}

[1] C. Li, Basis CAD of CAD, CAM/CAE, 2004, 560 p.
[2] J.F. Mamedov, "Development of structure of interface of complex automated planning of the technical systems software", Announcer of computer and informative technologies, № 5(107), 2013, pp. 18- 21.

[3] В.Н.Хализаев, Д.В. Угрюмов, Математическая модель принятия решений системного интегрирования в условиях множественного

[4] В.К. Урсаки, «Модели выбора инвестиционных проектов в условиях неопределенности и риска», Вестник Новгородского Государственного Университета им. Ярослава Мудрого, №74. 